\title{
Scanning Electrochemical Cell Microscopy Platform with Local Electrochemical Impedance Spectroscopy
}

Lei Cheng ${ }^{1,2}$, Rong Jin ${ }^{3}$, Dechen Jiang ${ }^{3 *}$, Jian Zhuang ${ }^{1,2^{*}}$, Xiaobo Liao ${ }^{1,2,4}$, Qiangqiang Zheng ${ }^{1,2}$

1. Key Laboratory of Education Ministry for Modern Design Rotor-Bearing System, Xi'an Jiaotong University, Xi'an 710049, P. R. China

2. School of Mechanical Engineering, Xi' an Jiaotong University, Xi' an 710049, P. R. China

3. State Key Laboratory of Analytical Chemistry for Life Science, School of Chemistry and Chemical Engineering, Nanjing University, Nanjing, Jiangsu 210023, P. R. China

4. Key Laboratory of Testing Technology for Manufacturing Process, Ministry of Education, Southwest University of Science and Technology, Mianyang 621010, P. R. China

Corresponding Authors

*Dechen Jiang, Email: dechenjiang@nju.edu.cn

*Jian Zhuang, Email: zhuangjian@mail.xjtu.edu.cn

\section{Table of Contents}

Finite Element Method Model

Repeated LEIS Measurements and Kramers-Kronig Transformation

\section{Supplementary Figures and Tables}

Figure S1 SEM images of the pipette tip.

Figure S2 SEM image of the droplet array. Inset: SEM image of one droplet, the diameter is about $180 \mathrm{~nm}$.

Figure S3 Concentration perturbation profile of $\mathrm{Fe}(\mathrm{CN})_{6}^{3-}$ at different $\mathrm{AC}$ frequencies.

Figure S5 Schematic diagram of rotational axis symmetry of the FEM model.

Figure S8 SEM images of N-rGO prepared by drying GO dissolved in different aqueous solution.

Table S2 Equivalent circuit component parameters obtained from the fitting of experimental data in Figure 2.

Table S3 Equivalent circuit component parameters fitted from the simulation impedance spectra with different $k_{h}$.

Table S4 Equivalent circuit component parameters fitted from the simulation impedance spectra with different $k_{\text {wet }}$.

Table S5 Equivalent circuit component parameters fitted from the simulation impedance spectra with different $\theta$.

Table S6 Equivalent circuit component parameters fitted from the simulation impedance spectra with different $r_{i}$. 


\section{Finite Element Method Model}

The domain of FEM model is shown in Figure S5. The 3D geometry is rotationally symmetrical along the symmetric axis. The radius of the tip opening is $r_{i}$, the height of the droplet is $h$, the contact radius of the meniscus is $r_{w e t}$, the half cone angle of the pipette is $\theta$, and the length of the pipette is $l$. Since the size of the back end of the pipette relative to the tip is infinite, an infinite domain was defined in the back end.

Since the space limitation of the tip, it is necessary to consider the electric field distribution in the FEM model domain. In cylindrical coordinates, the local potential in the electrolyte can be expressed as Poisson equation:

$$
\nabla^{2} \emptyset=\frac{\partial^{2} \emptyset}{\partial r^{2}}+\frac{1}{r} \frac{\partial \emptyset}{\partial r}+\frac{\partial^{2} \emptyset}{\partial z^{2}}=-\frac{\rho}{\varepsilon_{0} \varepsilon_{r}}
$$

where $\varnothing$ represents the electric potential, $\varepsilon_{0}$ refers to the permittivity of the vacuum, $\varepsilon_{r}$ represents the local dielectric constant, and $\rho$ is the local charge density. According to the Stern theory, there is a compact layer on the interface between the working electrode and the solution, which is called the outer Helmholtz plane (OHP). Hypothesis the thickness of the compact domain is $\mu$. Since there is no specific adsorption occurs, the compact domain contains tightly arranged adsorbed ions and solvent molecules. Hence, in the range of $0<z<\mu$, the local charge density is $\rho=0$. In the range of the diffusion layer $(z>\mu)$, the local charge density can be expressed by ion concentrations:

$$
\rho=\sum z_{i} c_{i} F
$$

where $z_{i}$ represents the charge number of specie $i, c_{i}$ represents the concentration of specie $i$, and $F$ is Faraday constant.

It worth noting that the electric field strength in the inside and outside of OHP has a huge difference. Therefore, the dielectric constant should not be considered as a single value under different electric field strength. The relationship between the dielectric constant with electric field strength can be expressed as follow ${ }^{1}$ :

$$
\varepsilon_{r}=n_{D}^{2}+\frac{7 N_{0} \mu_{v}\left(n_{D}^{2}+2\right)}{3 \varepsilon_{0} \sqrt{73} \varnothing} L\left[\frac{\sqrt{73} \emptyset \mu_{v}\left(n_{D}^{2}+2\right)}{6 k_{B} T}\right]
$$

where the function $L(x)$ can be expressed as $L(x)=\operatorname{coth}(x)-\frac{1}{x}, k_{B}$ is the Boltzmann constant, $n_{D}$ is the number of molecules per unit volume, $\mu_{v}$ is the dipole moment of the water molecule. $n_{D}$ and $\mu_{v}$ are set to 1.33 and $6.7380 \times 10^{-30} \mathrm{C} \cdot \mathrm{m}$.

In the regions outside the OHP, the concentration of species can be calculated by the NernstPlanck equation. Because diffusion and electromigration are the main components, the effect of convection in the solution on the material transfer can be ignored. The material transfer rate can be expressed as:

$$
\begin{gathered}
\frac{\partial c_{i}}{\partial t}=\nabla\left(-D_{i} \nabla c_{i}-z_{i} \mu_{i} c_{i} F \nabla \emptyset\right)=-D_{i}\left(\frac{\partial^{2} c_{i}}{\partial r^{2}}+\frac{1}{r} \frac{\partial c_{i}}{\partial r}+\frac{\partial^{2} c_{i}}{\partial z^{2}}\right)-\frac{1}{r} \frac{\partial}{\partial r}\left(r z_{i} D_{i} c_{i} \frac{F}{R T} \nabla \emptyset\right)- \\
\frac{\partial}{\partial z}\left(z_{i} D_{i} c_{i} \frac{F}{R T} \nabla \emptyset\right)
\end{gathered}
$$

where $D_{i}$ represents the diffusion coefficient of specie $i, \mu_{i}$ refers to the ionic mobility of specie $i$, and the ionic mobility can be represented as $\mu_{i}=\frac{D_{i}}{R T}$. The solution contains a total of four ions, so the diffusion coefficient is $D_{F e(C N)_{6}^{3-}}=D_{F e(C N)_{6}^{4-}}=1 \times 10^{-9} \mathrm{~m}^{2} / \mathrm{s}, D_{K^{+}}=D_{C l^{-}}=2 \times$ 
$10^{-9} \mathrm{~m}^{2} / \mathrm{s}$. The absolute temperature $\mathrm{T}$ is set to $298 \mathrm{~K}$.

A one-step redox reaction involving one-electron exchange occurs on the OHP can be expressed as a reversible (Nernstian) process:

$$
\mathrm{Fe}(\mathrm{CN})_{6}^{3-}+\mathrm{e}^{-} \rightleftharpoons \mathrm{Fe}(\mathrm{CN})_{6}^{4-}
$$

The reaction rate $(v)$ can be calculated according to the Butler-Volmer equation:

$$
v=k_{0}\left[C_{\text {red }} \exp \left(\alpha \frac{F}{R T}\left(E-E^{\prime}\right)\right)-C_{o x} \exp \left(-(1-\alpha) \frac{F}{R T}\left(E-E^{\prime}\right)\right)\right]
$$

where $C_{r e d}$ and $C_{o x}$ are the concentrations of oxides and reductants at OHP, $\alpha$ represents the transfer coefficient, $E$ represents the electrode potential, $E^{\prime}$ represents the standard potential, and $k_{0}$ represents standard rate constant. For the reaction (5), the standard rate constant is set to $k_{0}=$ $0.01 \mathrm{~cm} / \mathrm{s}$.

The boundary conditions of FEM model are listed in Table S1. The concentrations of all species at boundary face $\mathrm{A}$ are the same as the bulk solution far away from the pipette tip, so the concentrations condition at face A are $c_{\mathrm{Fe}(\mathrm{CN})_{6}^{3-}}=c_{\mathrm{Fe}(\mathrm{CN})_{6}^{4-}}=10 \mathrm{mM}, c_{\mathrm{K}^{+}}=c_{\mathrm{Cl}^{-}}=0.5 \mathrm{M}$. the electric potential at Face $\mathrm{A}$ is the same as the bulk solution, $\varnothing=0$. The inner wall of the capillary is negatively charged, and the surface charge density $(\sigma)$ of the inner wall of the pipette (Face B) is $-15 \mathrm{mC} / \mathrm{m}^{2}$. The capacitance of double layer $(C)$ on OHP (face C) is set to $0.35 \mathrm{~F} / \mathrm{m}^{2}$. The fluxes of redox reaction through OHP (Face $\mathrm{C}$ ) depends on the reaction rate $(v)$. The amplitude of the harmonic bias perturbation $\left(V_{a c}\right)$ is set to $50 \mathrm{mV}$. At the electrode surface (Face D), the electric potential of is the same as DC bias voltage.

\section{Repeated LEIS Measurements and Kramers-Kronig Transformation}

Figure S6a is the Nyquist plots of 100 repeated measurements over a single point of ITO electrode. As shown in Figure S2, the diameter of the point is about $180 \mathrm{~nm}$. The root mean square relative error of all LEIS data is about 3\%. It confirms that the results have good repeatability during the $8 \mathrm{~h}$ measurement. One set of LEIS results was evaluated by Kramers-Kronig transformation (Figure S6b). The sum of squared errors of the Kramers-Kronig transformation is about $0.3 \%$, indicating that the test results have a good causal relationship. 
Figure S1 SEM images of the pipette tip. (a) Top view of the orifice. (b) Side view of the tip.
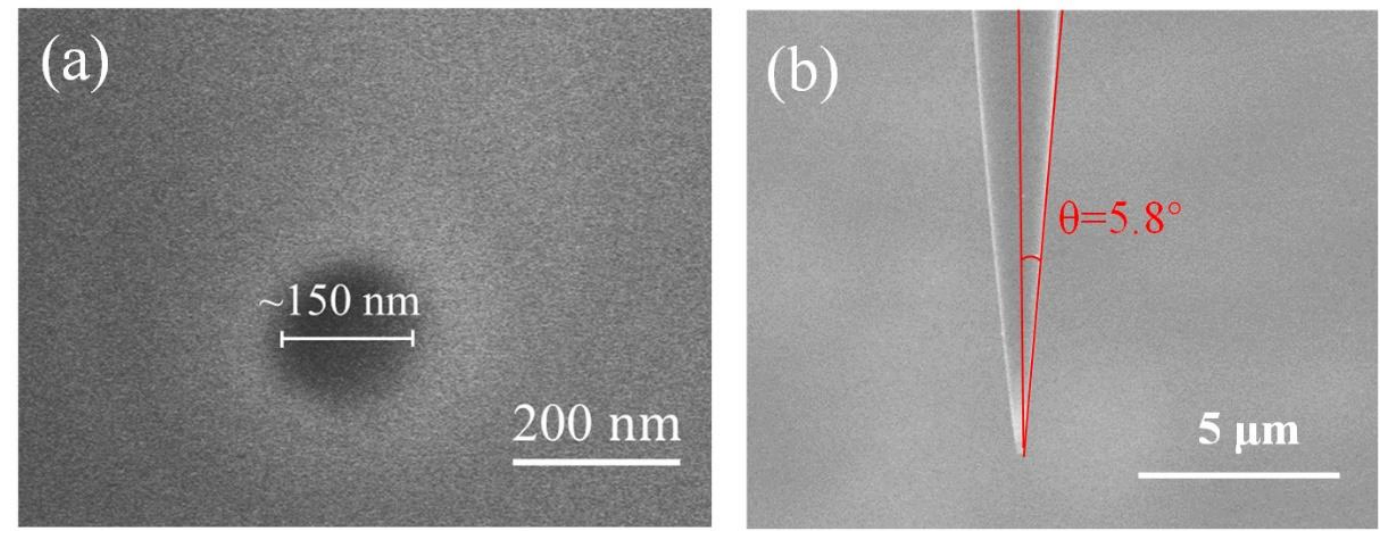

Figure S2 SEM image of the droplet array. Inset: SEM image of one droplet, the diameter is about $180 \mathrm{~nm}$.

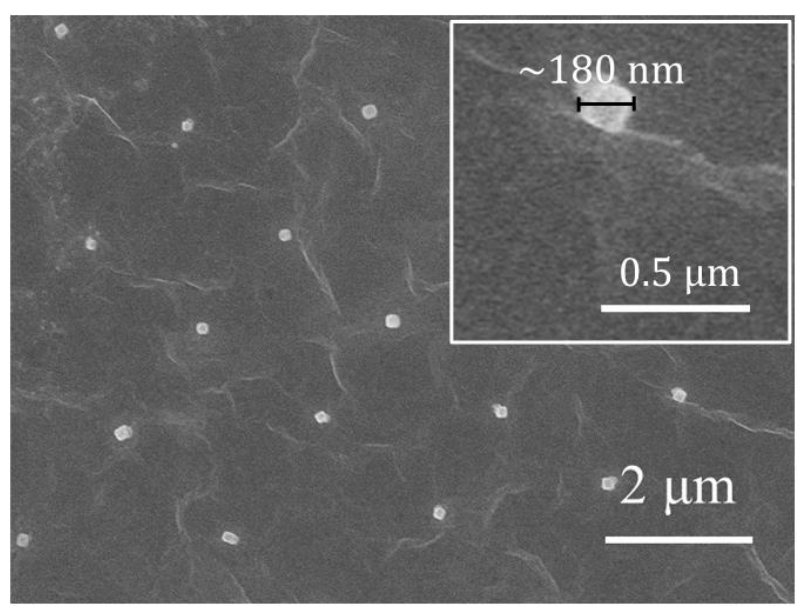


Figure $\mathrm{S} 3$ Concentration perturbation profile of $\mathrm{Fe}(\mathrm{CN})_{6}^{3-}$ at different $\mathrm{AC}$ frequencies.

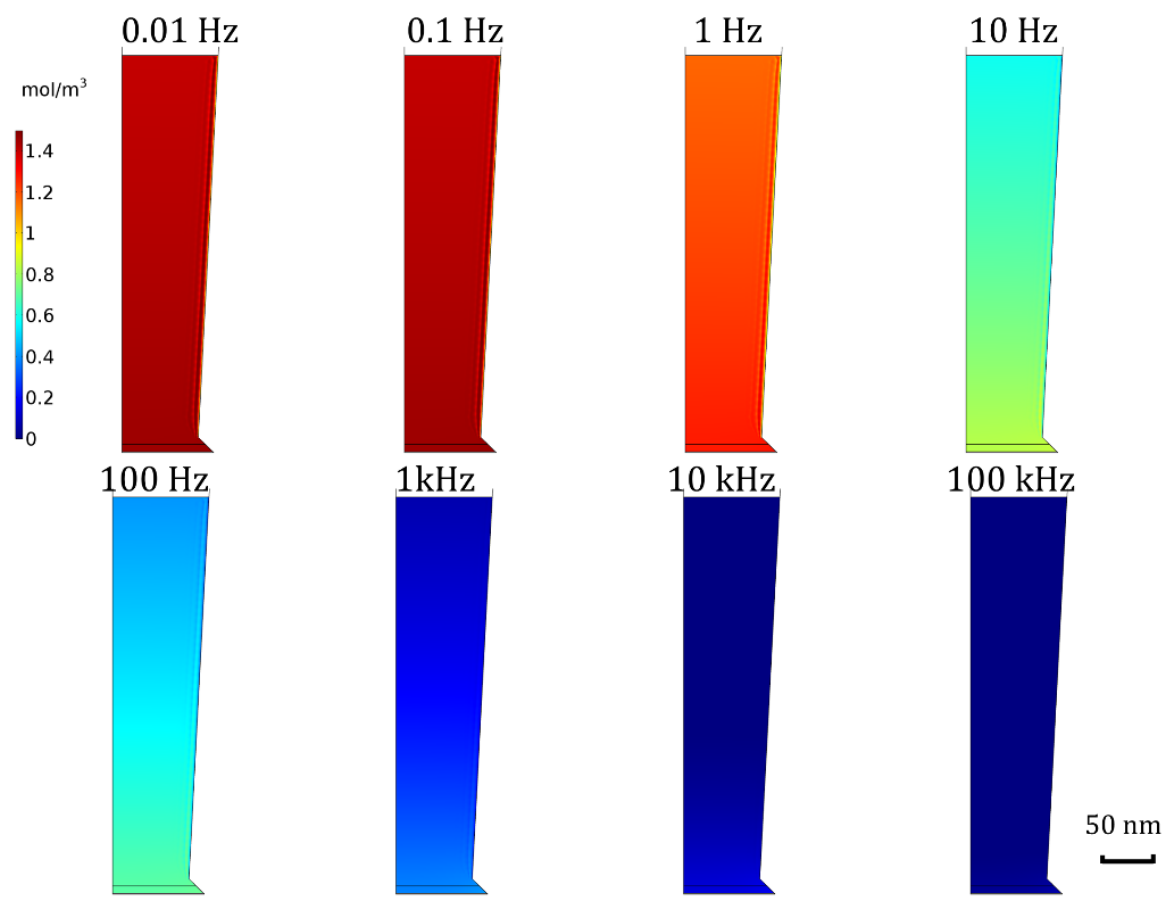

Figure S4 (a) Optical photograph and (b) SEM of the N-rGO surface

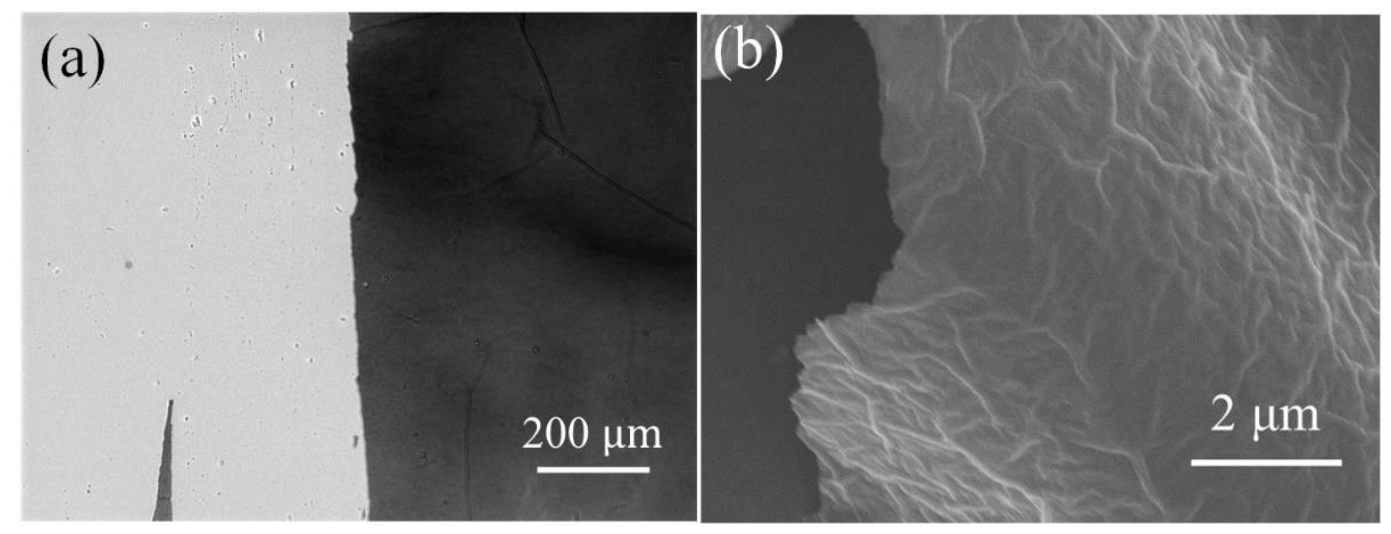


Figure S5 Schematic diagram of rotational axis symmetry of the FEM model. (a) The schematic diagram and size (not to scale); (b) simulation domain with grid, the area near the tip of the pipette uses a fine grid.

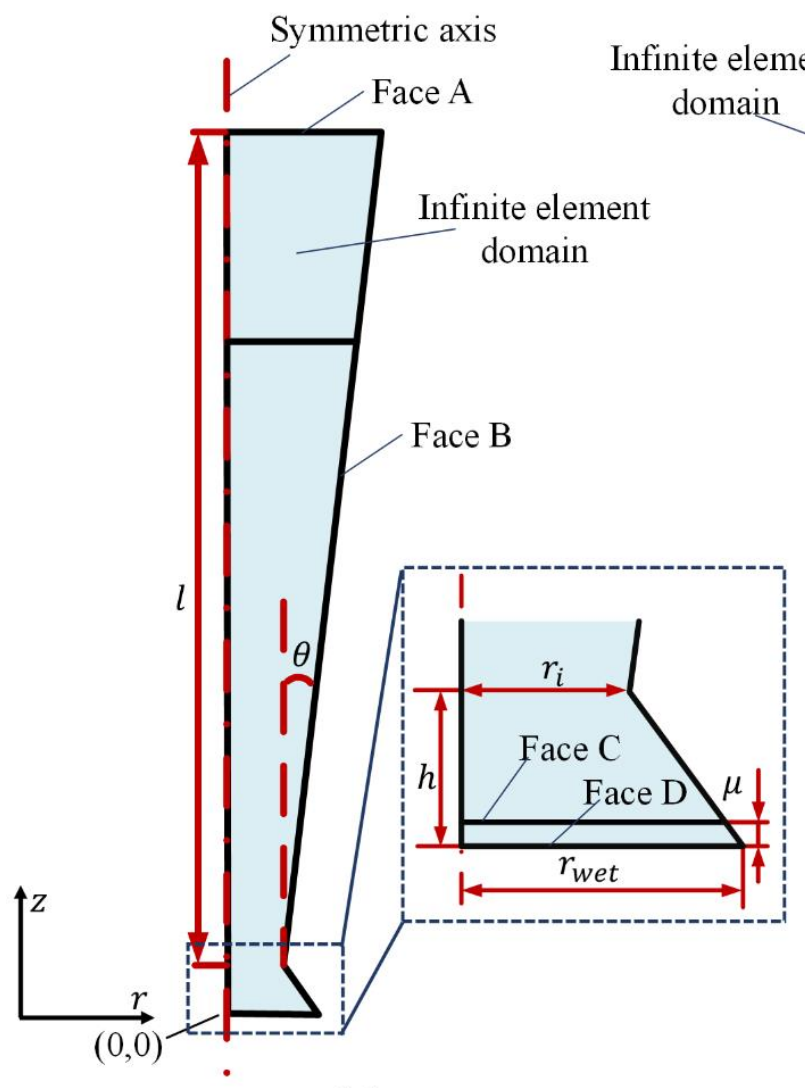

(a)

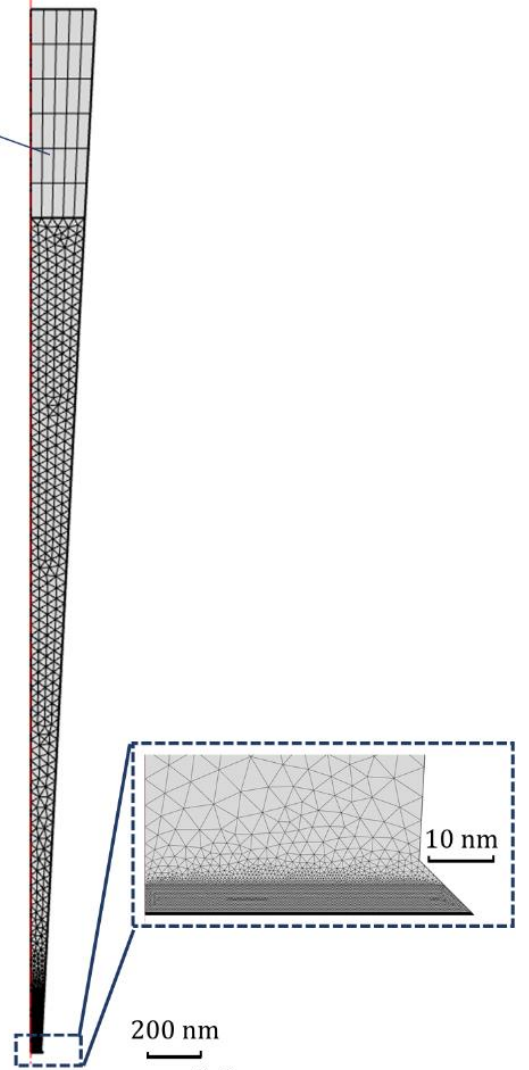

(b)

Figure S6 Stability and reliability test results. (a) Nyquist plots of 100 repeated LEIS measurements on single point at ITO electrode. The insert plot in (a) is the Nyquist diagram of high frequency domain. (b) Kramers-Kronig transformation from one set of experimental impedance spectra in (a). The frequency range of $\mathrm{AC}$ bias perturbation is $1 \mathrm{~Hz}-100 \mathrm{kHz}$.
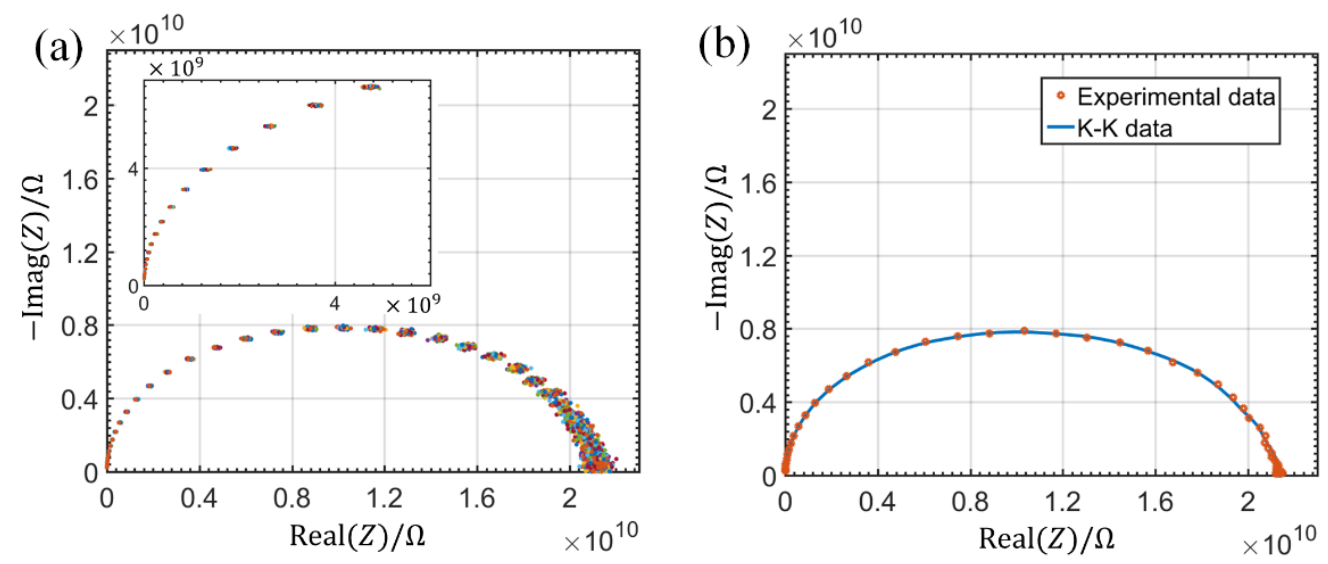
Figure S7 Confocal Raman spectrum result of N-rGO surface and edge.

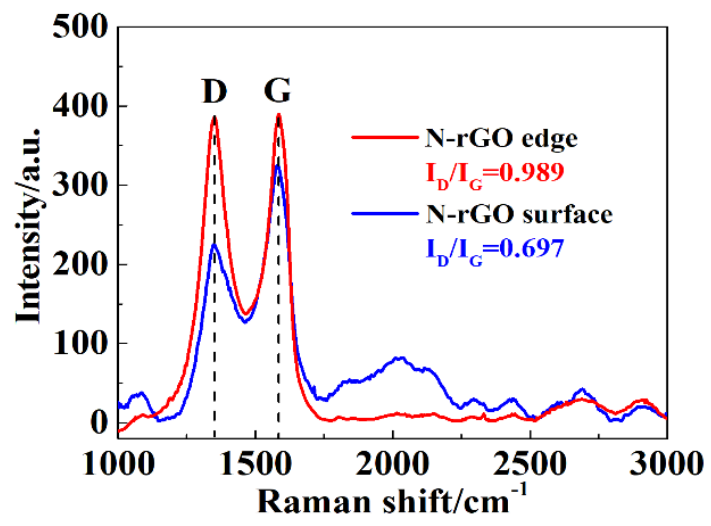

Figure S8 SEM images of N-rGO prepared by drying GO dissolved in (a) pure water and (b) half water and half alcohol.
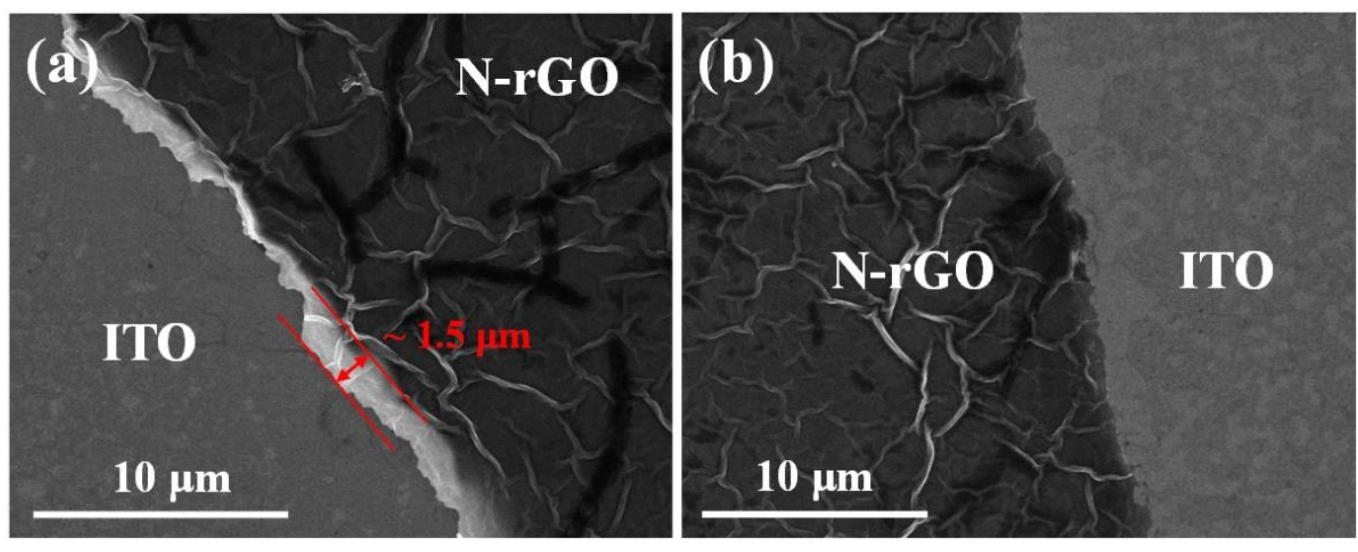
Table S1 The boundary conditions of FEM model.

\begin{tabular}{c|cc}
\hline Boundary & Electrostatics & Electroanalysis \\
\hline Face A & $\emptyset=0$ & $c_{i}=c_{i}^{b u l k}$ \\
Face B & $\sigma=-15 \mathrm{mC} / \mathrm{m}^{2}$ & $j_{i}=0$ \\
Face C & - & $C=0.35 \mathrm{~F} / \mathrm{m}^{2}, V_{a c}=50 \mathrm{mV}$, \\
& & $j_{F e(C N)_{6}^{3-}}=-j_{F e(C N)_{6}^{4-}}=v, j_{K^{+}}=j_{C l^{-}}=0$ \\
Face D & $\emptyset=E$ & - \\
\hline
\end{tabular}

Table S2 Equivalent circuit component parameters obtained from the fitting of experimental data in Figure 2.

\begin{tabular}{c|cccccc}
\hline Diameter & $R_{\text {bulk }} / \Omega$ & $R_{t} / \mathrm{G} \Omega$ & $C_{d l} / \mathrm{pF}$ & $R_{d} / \mathrm{G} \Omega$ & $T_{d} / \mathrm{ms}$ & $P_{d}$ \\
\hline $150 \mathrm{~nm}$ & 16490 & 11.2 & 0.00701 & 10.2 & 1.1 & 0.41 \\
$1000 \mathrm{~nm}$ & 8055 & 0.35 & 0.39 & 1.7 & 30.2 & 0.409 \\
\hline
\end{tabular}

Table S3 Equivalent circuit component parameters fitted from the simulation impedance spectra with different $k_{h}$.

\begin{tabular}{c|cccccc}
\hline$k_{h}$ & $R_{\text {bulk }}$ & $R_{t} / \mathrm{G} \Omega$ & $C_{d l} / \mathrm{pF}$ & $R_{d} / \mathrm{G} \Omega$ & $T_{d} / \mathrm{ms}$ & $P_{d}$ \\
\hline 0.1 & 251690 & 17.1 & $8.95 \times 10^{-3}$ & 16.9 & 3.42 & 0.38 \\
0.2 & 256560 & 17.2 & $8.95 \times 10^{-3}$ & 17.6 & 3.41 & 0.38 \\
0.3 & 256520 & 17.2 & $8.95 \times 10^{-3}$ & 17.9 & 3.40 & 0.38 \\
0.4 & 253530 & 17.1 & $8.95 \times 10^{-3}$ & 18.2 & 3.39 & 0.38 \\
0.5 & 248110 & 17.1 & $8.95 \times 10^{-3}$ & 18.4 & 3.38 & 0.38 \\
\hline
\end{tabular}

Table S4 Equivalent circuit component parameters fitted from the simulation impedance spectra with different $k_{\text {wet }}$.

\begin{tabular}{c|cccccc}
\hline$k_{\text {wet }}$ & $R_{\text {bulk }}$ & $R_{t} / \mathrm{G} \Omega$ & $C_{d l} / \mathrm{pF}$ & $R_{d} / \mathrm{G} \Omega$ & $T_{d} / \mathrm{ms}$ & $P_{d}$ \\
\hline 0.8 & 427610 & 25.41 & $3.97 \times 10^{-3}$ & 12.19 & 3.35 & 0.38 \\
0.9 & 387460 & 22.73 & $5.03 \times 10^{-3}$ & 13.59 & 3.37 & 0.38 \\
1 & 354880 & 20.54 & $6.21 \times 10^{-3}$ & 14.96 & 3.38 & 0.38 \\
1.1 & 328060 & 18.72 & $7.52 \times 10^{-3}$ & 16.29 & 3.40 & 0.38 \\
1.2 & 305800 & 17.18 & $8.95 \times 10^{-3}$ & 17.56 & 3.41 & 0.38 \\
1.3 & 287720 & 15.88 & $1.05 \times 10^{-2}$ & 18.75 & 3.42 & 0.38 \\
1.4 & 272860 & 14.76 & $1.22 \times 10^{-2}$ & 19.85 & 3.43 & 0.38 \\
1.5 & 260840 & 13.81 & $1.4 \times 10^{-2}$ & 20.88 & 3.43 & 0.39 \\
\hline
\end{tabular}


Table S5 Equivalent circuit component parameters fitted from the simulation impedance spectra with different $\theta$.

\begin{tabular}{c|cccccc}
\hline$\theta /^{\circ}$ & $R_{\text {bulk }}$ & $R_{t} / \mathrm{G} \Omega$ & $C_{d l} / \mathrm{pF}$ & $R_{d} / \mathrm{G} \Omega$ & $T_{d} / \mathrm{ms}$ & $P_{d}$ \\
\hline 3 & 305880 & 17.18 & $8.95 \times 10^{-3}$ & 17.56 & 3.41 & 0.38 \\
4 & 320510 & 13.27 & $8.94 \times 10^{-3}$ & 11.3 & 2.66 & 0.37 \\
5 & 324070 & 10.7 & $8.93 \times 10^{-3}$ & 7.81 & 2.12 & 0.36 \\
6 & 321160 & 8.96 & $8.93 \times 10^{-3}$ & 5.73 & 1.72 & 0.35 \\
7 & 314520 & 7.74 & $8.93 \times 10^{-3}$ & 4.41 & 1.43 & 0.34 \\
8 & 305910 & 6.87 & $8.93 \times 10^{-3}$ & 3.53 & 1.20 & 0.33 \\
9 & 295580 & 6.22 & $8.92 \times 10^{-3}$ & 2.91 & 1.02 & 0.33 \\
10 & 284780 & 5.72 & $8.92 \times 10^{-3}$ & 2.46 & 0.87 & 0.32 \\
\hline
\end{tabular}

Table S6 Equivalent circuit component parameters fitted from the simulation impedance spectra with different $r_{i}$.

\begin{tabular}{c|cccccc}
\hline$r_{i} / \mathrm{nm}$ & $R_{\text {bulk }}$ & $R_{t} / \mathrm{G} \Omega$ & $C_{d l} / \mathrm{pF}$ & $R_{d} / \mathrm{G} \Omega$ & $T_{d} / \mathrm{ms}$ & $P_{d}$ \\
\hline 50 & 781860 & 42.33 & $3.98 \times 10^{-3}$ & 25.64 & 1.56 & 0.41 \\
75 & 305880 & 17.18 & $8.95 \times 10^{-3}$ & 17.56 & 3.41 & 0.38 \\
100 & 11630 & 9.32 & $1.59 \times 10^{-2}$ & 13.63 & 6.06 & 0.37 \\
200 & 15200 & 2.21 & $6.33 \times 10^{-2}$ & 7.72 & 25.47 & 0.35 \\
300 & 11000 & 1.39 & $9.85 \times 10^{-2}$ & 8.04 & 58.75 & 0.35 \\
400 & 90443 & 0.78 & $1.75 \times 10^{-1}$ & 6.39 & 104.08 & 0.35 \\
500 & 91000 & 0.35 & $3.92 \times 10^{-1}$ & 3.71 & 160.14 & 0.35 \\
\hline
\end{tabular}

\section{References}

1 Liu, Y.; He, R.; Zhang, Q.; Chen, S. Theory of electrochemistry for nanometer-sized disk electrodes. The Journal of Physical Chemistry C, 2010, 114(24), 10812-10822. 